\title{
Is Less Invasive Surfactant Administration a Beneficial Method for Late Preterm Infants?
}

\author{
Mehmet Tekin, $\mathrm{MD}^{1 *}$; Musa Silahli, $\mathrm{MD}^{2}$; Zeynel Gokmen, $\mathrm{MD}^{2}$ \\ ${ }^{1}$ Department of Pediatrics, Faculty of Medicine, Baskent University, Konya, Turkey \\ ${ }^{2}$ Department of Pediatrics, Division of Neonatology, Faculty of Medicine, Baskent University, Konya, Turkey
}

\begin{abstract}
Background: Late preterm infants (LPIs) have increased steadily in all newborns delivery and they are the largest patient group requiring admission to the neonatal intensive care unit. Surfactant treatment is frequently used in LPIs in case of respiratory distress, but the procedure and the timing of surfactant administration are not well-known.

Objective: We aimed to evaluate the effect of surfactant administration techniques on pulmonary outcomes in LPIs with respiratory distress.

Methods: In this retrospective study, we compared the effects of the less invasive surfactant administration (LISA) technique and conventional treatment on respiratory and other morbidities in LPIs who have respiratory difficulties. We named these two groups as the LISA group and the conventional group (CG). Comparison of the mechanical ventilation (MV) rates between the groups was the primary outcome of our study.

Results: There were $25 \mathrm{LPIs}$ in each group. The duration of nasal continuous positive airway pressure (CPAP) and oxygenation were similar in both groups. The rate of MV and the duration of MV $(P=0.004$ and $P=0.02)$ were lower in the LISA group. Also, the need for more than 1 dose of surfactant was higher in the MV requiring group, although it was not statistically significant between the groups $(P=0.21)$.

Conclusion: Using the LISA technique for surfactant instillation reduces any MV requirement. LISA is a very useful and reliable technique in experienced hands in LPIs as in very preterm infants.

Keywords: Premature birth, Pulmonary surfactants, Ventilation

Cite this article as: Tekin M, Silahli M, Gokmen Z. Is less invasive surfactant administration a beneficial method for late preterm Infants? Arch Iran Med. 2021;24(10):759-764. doi: 10.34172/aim.2021.112
\end{abstract}

Received: April 21, 2020, Accepted:December 27, 2020, ePublished: October 1, 2021

\section{Introduction}

Late preterm infants (LPI), who are born between $34^{0 / 6}$ to $37^{6 / 7}$ weeks of gestational age, have increased steadily in all newborns delivery. They constitute the largest patient group who need admission to the neonatal intensive care unit. ${ }^{1,2}$ LPI hospitalizations constitute $8 \%$ of all newborns in the USA, ${ }^{1}$ and $10.6 \%$ in Turkey, respectively. ${ }^{3}$ The increase in advanced age pregnancy, multiple pregnancies along with the use of induction of labor, and cesarean sections are the main reasons for the rise of LPIs. ${ }^{4}$

The mortality rate and morbidity are known to increase in late preterm births compared to term births. The most common morbidity in these infants include: feeding problems, hypoglycemia, and jaundice..$^{5}$ Also, it has been shown in previous studies that LPIs experience neonatal respiratory diseases including respiratory distress syndrome (RDS) and transient tachypnea of the newborn (TTN), more frequently than term infants. ${ }^{5,6}$ Surfactant and antioxidant systems are not sufficiently developed in LPIs because they were born in the late saccular phase of lung development. ${ }^{7,8}$ Respiratory diseases may occur as a result of the absence of surfactant, poor gas exchange, and deferred intrapulmonary fluid absorption in the immature lung.
Surfactant treatment is the fundamental treatment used to improve respiratory failure in LPIs. Studies in LPIs have demonstrated the effectiveness of surfactant particularly in RDS, but the long-term benefit on early-onset pneumonia (EOP) was unclear. ${ }^{9-11}$

Surfactant treatment is frequently used in LPIs in case of respiratory failure, but the procedure and the timing of surfactant administration are not well-known. Less invasive surfactant administration (LISA), which is a newer technique for surfactant application, has been used as an alternative to conventional techniques and reduced the frequency of mechanical ventilation (MV) exposure. ${ }^{12-15}$ It is a basic and efficient method, and it is remote from major complications. ${ }^{9}$ In this retrospective study, we aimed to evaluate the effect of surfactant administration techniques on pulmonary outcomes in LPIs with respiratory distress.

\section{Materials and Methods Study Design}

This was a single-center retrospective observational study. We included late preterm neonates $\left(34^{0 / 6}\right.$ to $37^{6 / 7}$ gestational weeks) who were admitted to the NICU due to respiratory failure in the last ten years. The presence of 
major congenital malformations, chromosomal disorders, and inherited metabolic diseases were exclusion criteria. In 10 years of the medical records, 108 infants with respiratory difficulties were enrolled, 58 of whom were given surfactant therapy. After the exclusion of the cases, both groups included 25 eligible infants. The infants in the LISA group were involved especially the last 5-year period because non-invasive surfactant applications like LISA were applied more intensely. The flow diagram of the patients is shown in Figure 1. Clinical data and demographic information were gathered by reviewing the medical records of the enlisted infants.

The diagnoses of respiratory disorders were made by the following definitions. Respiratory failure was diagnosed by evaluating the clinical signs and chest radiographs. The clinical signs of the respiratory failure were described as follows: requirement for oxygen supplementation, tachypnea, intercostal retractions, and grunting. RDS was diagnosed as follows: the presence of the previously mentioned respiratory failure signs ${ }^{16}$; increased oxygen requirement during the initial 24 hours of life; the presence of diminished lung air content, reticulogranular pattern of the lungs, and air bronchograms in the chest radiograph; and the exclusion of other causes of respiratory failure..$^{17,18}$ Patients who needed oxygen supplement in the first 6 hours of life and then had a decrease in oxygen need during the following 18 hours, with near normal chest X-ray were identified as TTN. ${ }^{17}$ EOP was characterized as respiratory distress showing up within 72 hours of birth, ${ }^{19,20}$ with radiological features of pneumonia, with at least one risk factor for infection (maternal fever $>38^{\circ} \mathrm{C} /$ prolonged rupture of membranes $>18$ hours/maternal urinary tract

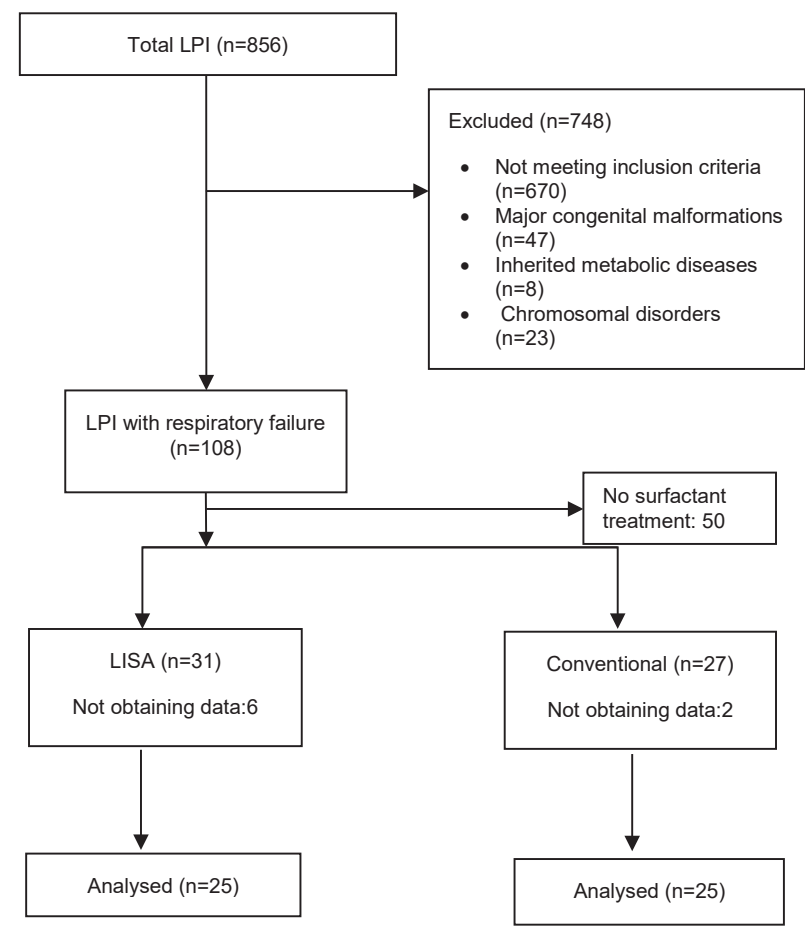

Figure 1. Flow Diagram. infection/suspected or confirmed chorioamnionitis/ spontaneous preterm labor) or neonatal blood culture positive for a proven pathogen. If the meconium-stained newborn had respiratory distress with demonstrated aspiration in the chest radiography, meconium aspiration syndrome was diagnosed. ${ }^{17}$

Examined data included gender, birth weight, gestational age, multiple births, mode of delivery, small for gestational age (SGA), duration of NICU and hospital stay, and respiratory pathologies. The analyzed maternal factors including antenatal steroid administration, maternal diabetes, placental abruption, hypertensive disorders, prolonged premature rupture of membranes $(\mathrm{pPROM})>18 \mathrm{~h}$, and clinical chorioamnionitis were obtained from the medical records.

Gestational age was calculated according to the ultrasound follow-up and the last menstrual period of the mother. Newborns, whose birth weights were below the 10th percentile for gestational age, were defined as SGA. Clinical chorioamnionitis was characterized as the presence of fever with at least one of the accompanying findings: maternal leukocytosis $>15000 / \mathrm{mm}^{3}$, uterine tenderness, fetal tachycardia, or foul-smelling amniotic fluid. The patients were followed up until they met the discharge criteria. Healthy infants, who did not receive additional treatment, were discharged when it was observed that they could get adequate care from their mother.

We recorded the following data for each infant who was treated with surfactant: Conventional or LISA procedure for surfactant therapy; the number of surfactant doses; age (days of life) at first administration of surfactant; duration of noninvasive respiratory support [nasal continuous positive airway pressure (nCPAP)]; need for and duration of $\mathrm{MV}$; duration of oxygen administration; need for inhaled nitric oxide (iNO) and high-frequency oscillatory ventilation (HFOV); occurrence of pneumothorax; occurrence of persistent pulmonary hypertension of the newborn (PPHN) and patent ductus arteriosus (PDA) that required pharmacological treatment (diagnosed by heart ultrasound); occurrence of intraventricular hemorrhage (IVH), periventricular leukomalacia (PVL), necrotizing enterocolitis (NEC) requiring surgery, proven sepsis (early-onset sepsis: 3 days of life; late-onset sepsis $>3$ days of life); and mortality.

\section{Respiratory Management}

All infants were initially managed with nCPAP or oxygen treatment. Surfactant via intratracheal route was administered to patients who needed fraction of inspired oxygen $\left(\mathrm{FiO}_{2}\right)$ of $35 \%$ and above at $6 \mathrm{~cm} \mathrm{H} \mathrm{H}_{2} \mathrm{O}$ pressure in nCPAP to achieve target saturation. The first dose of surfactant (Curosurf, Chiesi Farmaceutici, Parma, Italy) was $200 \mathrm{mg} / \mathrm{kg}$, followed by $100 \mathrm{mg} / \mathrm{kg}$ as needed according to the clinical guidelines. Initiation criteria for MV were as follows: $\mathrm{pH}<7.20$ with $\mathrm{PaCO} 2>65 \mathrm{~mm} \mathrm{Hg}$, 
or $\mathrm{PaO} 2<50 \mathrm{~mm} \mathrm{Hg}$ with $\mathrm{FiO}_{2} \geq 50 \%$, or if infants had frequent episodes of apnea in spite of sufficient nCPAP support and oxygenation. Infants had to meet all of the following criteria before being extubated: $\mathrm{FiO}_{2}<40 \%$, $\mathrm{PaCO}_{2}<65 \mathrm{~mm} \mathrm{Hg}$ with $\mathrm{pH}>7.20$, mean airway pressure $(\mathrm{MAP})<7 \mathrm{cmH}_{2} \mathrm{O}$, and hemodynamic stability.

\section{LISA Application}

LISA has been a frequently used method in our NICU in the last five years. It was applied as described below. First, the patient was positioned as appropriate. The catheter length was not shortened. A $5 \mathrm{~F}$ or $6 \mathrm{~F}$ catheter was pushed forward through the vocal cords under direct vision using a laryngoscope, without the requirement for Magill's forceps and with no sedation. Then, gentle pressure was applied on the trachea to avoid reflux of the surfactant after the catheter passed through the vocal cords for $1 \mathrm{~cm}$. After catheter placement, the laryngoscope was removed. An appropriate poractant dose with an extra $1 \mathrm{~mL}$ of air was drawn up into the syringe. The purpose of drawing up the extra air was to eliminate the dead volume of the instillation catheter. In this position, a second member of the NICU staff administered the surfactant within 30 seconds. Then, the catheter was removed. Poractant was used in all of the patients.

\section{Conventional Method}

Surfactant administration was performed for infants in the CG after intubation. After intubation, a $5 \mathrm{~F}$ or $6 \mathrm{~F}$ catheter was passed through the tube. The catheter forward level was adjusted according to the tube length and the catheter tip was left $1-2 \mathrm{~cm}$ above the carina. In this position, a second member of the NICU staff administered the surfactant within 30 seconds. Then, the catheter was removed and gentle positive pressure ventilation was performed for approximately 30 seconds to ensure surfactant distribution. Poractant was used in all of the patients. An appropriate poractant dose with an extra $1 \mathrm{~mL}$ of air was drawn up into the syringe. The purpose of drawing up the extra air was to eliminate the dead volume of the instillation catheter. In the conventional group, the decision of continuing intubation was made as follows. If only surfactant administration was planned, the INSURE (INtubation-SURfactantExtubation) method was preferred. The other patients remained intubated. Extubation was planned according to the criteria mentioned above.

\section{Primary Outcomes}

The primary outcome of our study was to compare the need for MV between these two methods. We also evaluated the duration of nCPAP, MV, and oxygen administration; the duration of NICU and hospital stay; occurrence of pneumothorax and PPHN in the same population.

\section{Statistical Analysis}

Based on Olivier's study, the intubation rate after LISA would decrease by $85 \%$ to $30 \%$ (9). Post hoc power analyses showed that the study has over $90 \%$ power to detect a $55 \%$ difference in rates of MV in the LISA versus the CG group.

Descriptive statistics of scale variables included mean \pm standard deviation (SD) or median (range) as appropriate. Demographic and clinical continuous variables were compared using the 2- independent Student's $t$ test for normally distributed values and the Mann-Whitney U test for non-normally distributed values. $\mathrm{Z}$ scores of skewness, kurtosis, and Shapiro Wilk statistics were used to assess whether the continuous variables were normally distributed. Categorical variables were compared using Fisher's exact test. The univariate analysis to identify variables associated with MV requirement was conducted using chi-square, Fisher's exact, Student's $t$ test, and Mann-Whitney $U$ tests, where appropriate. For all tests, the level of statistical significance was set at $P=0.05$. SPSS 25 was used for all data analyses.

\section{Results}

During the 10 years, 856 LPIs were born in our hospital. The number of patients admitted to the NICU due to respiratory failure was $108(13 \%)$. Surfactant treatment was performed for 58 infants (8\% of the overall late preterm population). Surfactant was administered to 31 patients using the LISA method and 27 patients using the conventional method. When the cases with incomplete data were excluded, there remained 25 LPIs in each group (Figure 1).

The baseline characteristics are shown in Table 1. There were no significant differences between the two groups except for birth weight. Although there was no difference between the gestational weeks, the presence of SGA infants in the CG $(n=3)$ may have caused this circumstance.

Table 2 shows the respiratory outcomes. Infants who were treated with LISA had a lower rate of MV $(P=0.004)$ and duration of MV $(P=0.02)$. There was no difference between the groups in the duration of nCPAP and oxygenation. Although more than one dose of surfactant administration was more common in the CG group, the difference was not statistically significant $(P=0.21)$. The frequency of pneumothorax and HFOV use were equal in both groups ( $n=4$ and $n=2$, respectively). Also, there was no difference between the groups in frequency of PPHN, and duration of NICU and hospital stay. Although PPHN was detected in 6 cases in all of the infants, iNO use was not required in any of the patients. Secondary outcomes are given in Table 3 and there were no differences in the parameters between the groups.

\section{Discussion}

LPIs constitute the majority of neonatal intensive care admissions and they are likely to exhibit respiratory morbidities in long- and short-term periods. ${ }^{21}$ While there is a large number of studies in very preterm infants about surfactant applications, there are not enough studies in 
Table 1. Baseline Characteristics

\begin{tabular}{|c|c|c|c|}
\hline & LISA $(n=25)$ & CG $(n=25)$ & $P$ \\
\hline Birth weight, $g$, mean $\pm S D$ & $2792 \pm 400$ & $2448 \pm 505$ & 0.01 \\
\hline Gestational age, wk, median (min-max) & $36(34-37)$ & $35(34-37)$ & 0.19 \\
\hline Gender, male, n (\%) OR (95\% Cl): 0.49 (0.15-1.60) & $14(56 \%)$ & $18(72 \%)$ & 0.37 \\
\hline SGA, n (\%) & $0(0 \%)$ & $3(12 \%)$ & 0.23 \\
\hline Multipl birth, n (\%) & $0(0 \%)$ & $3(12 \%)$ & 0.23 \\
\hline C/S, n (\%) OR (95\% Cl): 1 (0.05-16.90) & $24(96 \%)$ & $24(96 \%)$ & 1.00 \\
\hline Antenatal steroid, $\mathrm{n}(\%)$ OR $(95 \% \mathrm{Cl}): 2.31(0.5-10.5)$ & $3(12 \%)$ & $6(24 \%)$ & 0.46 \\
\hline Maternal DM, n (\%) OR (95\% Cl): 1 (0.13-7.7) & $2(8 \%)$ & $2(8 \%)$ & 1.00 \\
\hline Placental abruption, n (\%) OR (95\% Cl): $0.3(0.03-3.1)$ & $3(12 \%)$ & $1(4 \%)$ & 0.61 \\
\hline PPROM, n (\%) OR (95\% Cl): 0.47 (0.04-0.6) & $2(8 \%)$ & $1(4 \%)$ & 1.00 \\
\hline Maternal hypertension, $\mathrm{n}(\%)$ OR $(95 \% \mathrm{Cl}): 0.63(0.09-4.1)$ & $3(12 \%)$ & $2(8 \%)$ & 1.00 \\
\hline RDS, n (\%) OR (95\% Cl): $0.6(0.2-1.8)$ & $14(56 \%)$ & $11(44 \%)$ & 0.57 \\
\hline TTN, n (\%) OR (95\% Cl): $1.5(0.4-5.7)$ & $5(20 \%)$ & $7(28 \%)$ & 0.74 \\
\hline EOP, n (\%) OR (95\% Cl): $1.2(0.3-4.3)$ & $6(24 \%)$ & $7(28 \%)$ & 1.00 \\
\hline First surfactant time, day, median (min-max) & $2(1-4)$ & $2(1-4)$ & 0.59 \\
\hline PDA, n (\%) OR (95\% Cl): $1(0.1-7.7)$ & $2(8 \%)$ & $2(8 \%)$ & 1.00 \\
\hline
\end{tabular}

There were no significant differences between the two groups except for birth weights which was associated with the SGA infant in the CG.

PPROM, Prolonged premature rupture of membranes; SGA, Small for gestational age; C/S, cesarean section; RDS: Respiratory distress syndrome; TTN, Transient tachypnea of the newborn; EOP, Early-onset pneumonia; CG, Conventional group; PDA, Patent ductus arteriosus.

Table 2. Respiratory Outcomes

\begin{tabular}{|c|c|c|c|c|}
\hline & LISA $(n=25)$ & CG $(n=25)$ & Effect Size $(95 \% \mathrm{Cl})$ & $\boldsymbol{P}$ \\
\hline $\begin{array}{l}\text { Two or more surfactant doses, } \mathrm{n}(\%), \text { OR }(95 \% \mathrm{Cl}): 2.66 \\
(0.75-9.45)\end{array}$ & $5(20 \%)$ & $10(40 \%)$ & $\begin{array}{c}\text { Cramer's } \vee \text { value }=0.218 \\
(0.210-0.226)\end{array}$ & 0.21 \\
\hline $\begin{array}{l}\text { MV requirement, } \mathrm{n}(\%) \\
\text { OR }(95 \% \mathrm{Cl}): 7.11(1.98-25.46)\end{array}$ & $5(20 \%)$ & $16(64 \%)$ & $\begin{array}{c}\text { Cramer's V value }=0.446 \\
(0.003-0.005)\end{array}$ & 0.004 \\
\hline Duration of MV, day, median (min-max) & $0(0-6)$ & $2(0-11)$ & $\begin{array}{c}0.184 \\
(0.01-0.03)\end{array}$ & 0.02 \\
\hline Duration of nCPAP, day, median (min-max) & $5(2-20)$ & $4(1-17)$ & $\begin{array}{c}0.031 \\
(0.212-0.228)\end{array}$ & 0.21 \\
\hline $\begin{array}{l}\text { Pneumothorax, n (\%) } \\
\text { OR }(95 \% \mathrm{Cl}): 1(0.2-4.5)\end{array}$ & $4(16 \%)$ & $4(16 \%)$ & $\begin{array}{c}\text { Cramer's } \vee \text { value }=0 \\
(1-1)\end{array}$ & 1.00 \\
\hline $\begin{array}{l}\text { HFOV, n (\%) } \\
\text { OR (95\% Cl): } 1(0.13-7.71)\end{array}$ & $2(8 \%)$ & $2(8 \%)$ & $\begin{array}{c}\text { Cramer's } \vee \text { value }=0 \\
(1-1)\end{array}$ & 1.00 \\
\hline $\begin{array}{l}\text { PPHN, n (\%) } \\
\text { OR (95\% Cl): } 2.19(0.36-13.2)\end{array}$ & $2(8 \%)$ & $4(16 \%)$ & $\begin{array}{c}\text { Cramer's } \vee \text { value }=0.123 \\
(0.658-0.676)\end{array}$ & 0.66 \\
\hline Duration of NICU stay, day, median (min-max) & $10(7-18)$ & $12(7-35)$ & $\begin{array}{c}0.041 \\
(0.142-0.168)\end{array}$ & 0.15 \\
\hline Duration of hospital stay, day, median (min-max) & $11(8-29)$ & $13(8-35)$ & $\begin{array}{c}0.07 \\
(0.05-0.06)\end{array}$ & 0.05 \\
\hline Duration of $\mathrm{O}_{2}$ administration, day, median (min-max) & $8(6-23)$ & $9(3-22)$ & $\begin{array}{c}0.012 \\
(0.432-0.452)\end{array}$ & 0.44 \\
\hline
\end{tabular}

$\mathrm{MV}$ requirement and duration of MV were significantly lower in the LISA group. There were no significant differences in other respiratory outcomes between the two groups.

LISA, less invasive surfactant administration; HFOV, High-Frequency Oscillation Ventilation; MV, Mechanical ventilation; nCPAP, Nasal continuous airway pressure; PPHN, Persistent Pulmonary hypertension; NICU, Neonatal intensive care unit; CG, Conventional group

Table 3. Secondary Outcomes

\begin{tabular}{|c|c|c|c|}
\hline & LISA $(n=25)$ & CG $(n=25)$ & $\boldsymbol{P}$ \\
\hline IVH, n (\%) OR (95\% CI): 1 (0.05-16.9) & $1(4 \%)$ & $1(4 \%)$ & 1.00 \\
\hline PVL, n (\%) OR (95\% Cl): 1 (0.05-16.9) & $1(4 \%)$ & $1(4 \%)$ & 1.00 \\
\hline Early sepsis, n (\%) & $0(0 \%)$ & $2(8 \%)$ & 0.49 \\
\hline Late onset sepsis, n (\%) OR (95\% Cl): 1 (0.05-16.9) & $1(4 \%)$ & $1(4 \%)$ & 1.00 \\
\hline Mortality, n (\%) & $1(4 \%)$ & $0(0 \%)$ & 1.00 \\
\hline
\end{tabular}

There was no statistically significant difference between the two groups in terms of secondary outcomes.

$\mathrm{IVH}$, Intraventricular hemorrhage; PVL, Periventricular leukomalacia 
LPIs. In a study in which 45 moderate-late preterms with RDS ( $32^{0 / 7}$ to $36^{6 / 7}$ ) were evaluated, it was shown that MV requirement decreased in the first 3 days with LISA, but the duration of MV did not change. ${ }^{9}$ In our study, we observed a decrease in requirement and duration of MV with LISA.

When the diagnoses of the infants were examined, it was found that $37 \%$ of them had RDS. This rate is similar to a previous study in which short-term respiratory outcomes were examined in LPIs. ${ }^{17}$ Although RDS is common in this population, other respiratory diseases also play an important role in NICU admission and surfactant may be required in these diseases due to secondary surfactant inactivation and dysfunction. ${ }^{22}$ Therefore, our study, which included all late preterms with respiratory distress, was more inclusive in predicting the effect of LISA use in respiratory diseases in LPIs.

When RDS rates are considered in LPIs, the rate of using antenatal steroids remains low. In a study by SürmeliOnay et $\mathrm{al}^{23}$ the antenatal steroid rate was $15.6 \%$. This rate was similarly $18 \%$ in our study. These rates may have been influenced by the policy of the perinatology unit. As in many centers, our perinatology unit administers antenatal steroid at 34 weeks and earlier. Antenatal steroid therapy to prevent the RDS after the $34^{\text {th }}$ week of pregnancy requires substantial evidence.

Another remarkable point in our study is cesarean rates. Only two cases $(8 \%)$ were born vaginally in the study population. In another study from Turkey including 77 LPIs, ${ }^{23}$ the vaginal delivery rate was founded to be $16.9 \%$. Cesarean section is high in this period of pregnancy because the majority of them are high-risk pregnancies. Even so, it should not be ignored that cesarean sections, especially elective cesareans, increase respiratory problems and risks in the infant should be taken into account when elective delivery is indicated.

In our study, we found that the rate of surfactant repeat in the LISA group was $20 \%$. The repeated surfactant administration rate in the LISA group was $37.5 \%$ in the study by Olivier and colleagues. ${ }^{9}$ The reason for this high rate in Olivier's study may be related to surfactant reflux. We applied gentle pressure on the trachea during surfactant administration and this may have probably helped to reduce surfactant reflux.

As mentioned above, the LISA method has some problems such as surfactant reflux, apnea, desaturation, and bradycardia. In our study, we did not examine these parameters in details, but we did not encounter any serious complications. A research article about LISA showed that up to $20 \%$ of cases of short periods of bradycardia and desaturation are observed. However, most of these complications were self-limiting and could be controlled with simple interventions. ${ }^{24}$

Two patients had to be intubated because of thoracic rigidity after fentanyl administration in Olivier's study. ${ }^{9}$ We do not use sedation in our LISA practice to avoid such complications. We do not have sufficient evidence for sedation and studies are needed on this subject.

Similar to a previous study, ${ }^{9}$ we did not find any difference regarding the duration of nCPAP, $\mathrm{O}_{2}$ administration, hospital stay, and NICU stay. In our study, one patient died and the reason for exitus was unrelated to respiratory problems. Sürmeli-Onay et $\mathrm{al}^{23}$ found that the severity of the underlying lung diseases and the existence of pulmonary hypertension contributed greatly to mortality. ${ }^{23}$ Improvement in the management of respiratory diseases may have caused this difference.

The strength of our study is that it was the first study to evaluate LISA in LPIs who were admitted to NICU with respiratory problems. The main limitation of the study is the retrospective structure and the small number of cases. Also, some of the post-intubation surfactant administration, which is accepted as the conventional method, was made with the INSURE method, but it could not be evaluated as a separate group due to the small number of cases. Another limitation of our study is that with the parameters obtained from univariate analyses, we aimed to create a model that predicts the need for MV; however, reasonable outcomes could not be obtained due to the small sample size of the study.

In conclusion, LISA reduces the need for and duration of $\mathrm{MV}$ in LPIs compared to the conventional method. Nevertheless, better structured prospective studies are needed in this area.

\section{Authors' Contribution}

MT: Data collection, manuscript writing. MS: Data collection, manuscript writing. ZG: Editing.

\section{Conflict of Interest Disclosures}

The authors have no conflicts of interest to declare.

\section{Ethical Statement}

Ethical approval was not required because of the retrospective structure.

\section{Funding Sources}

No funding was received for this study.

\section{References}

1. Shapiro-Mendoza CK, Lackritz EM. Epidemiology of late and moderate preterm birth. Semin Fetal Neonatal Med. 2012;17(3):120-5. doi: 10.1016/j.siny.2012.01.007.

2. Martin JA, Hamilton BE, Sutton PD, Ventura SJ, Menacker F, Kirmeyer S, et al. Births: final data for 2005. Natl Vital Stat Rep. 2007;56(6):1-103.

3. Celik IH, Demirel G, Canpolat FE, Dilmen U. A common problem for neonatal intensive care units: late preterm infants, a prospective study with term controls in a large perinatal center. J Matern Fetal Neonatal Med. 2013;26(5):459-62. doi: 10.3109/14767058.2012.735994.

4. Mally PV, Bailey S, Hendricks-Muñoz KD. Clinical issues in the management of late preterm infants. Curr Probl Pediatr Adolesc Health Care. 2010;40(9):218-33. doi: 10.1016/j. cppeds.2010.07.005.

5. Leone A, Ersfeld P, Adams M, Schiffer PM, Bucher HU, Arlettaz R. Neonatal morbidity in singleton late preterm infants compared with full-term infants. Acta Paediatr. 2012;101(1):e6-10. doi: 10.1111/j.1651-2227.2011.02459.x. 
6. Cheng YW, Kaimal AJ, Bruckner TA, Halloran DR, Caughey $A B$. Perinatal morbidity associated with late preterm deliveries compared with deliveries between 37 and 40 weeks of gestation. BJOG. 2011;118(12):1446-54. doi: 10.1111/j.14710528.2011.03045.x.

7. Kotecha S. Lung growth: implications for the newborn infant. Arch Dis Child Fetal Neonatal Ed. 2000;82(1):F69-74. doi: 10.1136/fn.82.1.f69.

8. Joshi S, Kotecha S. Lung growth and development. Early Hum Dev. 2007;83(12):789-94. doi: 10.1016/j. earlhumdev.2007.09.007.

9. Olivier F, Nadeau S, Bélanger S, Julien AS, Massé E, Ali N, et al. Efficacy of minimally invasive surfactant therapy in moderate and late preterm infants: a multicentre randomized control trial. Paediatr Child Health. 2017;22(3):120-4. doi: 10.1093/pch/pxx033.

10. Dani C, Mosca F, Vento G, Tagliabue P, Picone S, Lista G, et al. Effects of surfactant treatment in late preterm infants with respiratory distress syndrome. J Matern Fetal Neonatal Med. 2018;31(10):1259-66. doi: 10.1080/14767058.2017.1313828.

11. Deshpande S, Suryawanshi P, Ahya K, Maheshwari R, Gupta S. Surfactant therapy for early onset pneumonia in late preterm and term neonates needing mechanical ventilation. J Clin Diagn Res. 2017;11(8):SC09-SC12. doi: 10.7860/ jcdr/2017/28523.10520.

12. Göpel W, Kribs A, Ziegler A, Laux R, Hoehn T, Wieg C, et al. Avoidance of mechanical ventilation by surfactant treatment of spontaneously breathing preterm infants (AMV): an open-label, randomised, controlled trial. Lancet. 2011;378(9803):162734. doi: 10.1016/s0140-6736(11)60986-0.

13. Kribs A, Roll C, Göpel W, Wieg C, Groneck P, Laux R, et al. Nonintubated surfactant application vs conventional therapy in extremely preterm infants: a randomized clinical trial. JAMA Pediatr. 2015;169(8):723-30. doi: 10.1001/ jamapediatrics.2015.0504.

14. Kanmaz HG, Erdeve O, Canpolat FE, Mutlu B, Dilmen U.
Surfactant administration via thin catheter during spontaneous breathing: randomized controlled trial. Pediatrics. 2013;131(2):e502-9. doi: 10.1542/peds.2012-0603.

15. Dargaville PA, Aiyappan A, Cornelius A, Williams C, De Paoli AG. Preliminary evaluation of a new technique of minimally invasive surfactant therapy. Arch Dis Child Fetal Neonatal Ed. 2011;96(4):F243-8. doi: 10.1136/adc.2010.192518.

16. Rudolph AJ, Desmond MM, Pineda RG. Clinical diagnosis of respiratory difficulty in the newborn. Pediatr Clin North Am. 1966;13(3):669-92. doi: 10.1016/s0031-3955(16)31876-4.

17. Natile M, Ventura ML, Colombo M, Bernasconi D, Locatelli A, Plevani C, et al. Short-term respiratory outcomes in late preterm infants. Ital J Pediatr. 2014;40:52. doi: 10.1186/18247288-40-52.

18. Mortensson W, Noack G, Curstedt T, Herin P, Robertson B. Radiologic observations in severe neonatal respiratory distress syndrome treated with the isolated phospholipid fraction of natural surfactant. Acta Radiol. 1987;28(4):389-94.

19. Nissen MD. Congenital and neonatal pneumonia. Paediatr Respir Rev. 2007;8(3):195-203. doi: 10.1016/j. prrv.2007.07.001.

20. Reiterer F. Neonatal pneumonia. In: Resch B, ed. Neonatal Bacterial Infection. IntechOpen; 2013. doi: 10.5772/54310.

21. Kotecha SJ, Dunstan FD, Kotecha S. Long term respiratory outcomes of late preterm-born infants. Semin Fetal Neonatal Med. 2012;17(2):77-81. doi: 10.1016/j.siny.2012.01.004.

22. Finer NN. Surfactant use for neonatal lung injury: beyond respiratory distress syndrome. Paediatr Respir Rev. 2004;5 Suppl A:S289-97. doi: 10.1016/s1526-0542(04)90053-x.

23. Sürmeli-Onay O, Korkmaz A, Yiğit S, Yurdakök M. Surfactant therapy in late preterm infants: respiratory distress syndrome and beyond. Turk J Pediatr. 2012;54(3):239-46.

24. Herting E. Less invasive surfactant administration (LISA) - ways to deliver surfactant in spontaneously breathing infants. Early Hum Dev. 2013;89(11):875-80. doi: 10.1016/j. earlhumdev.2013.08.023. 